This Section of Epidemiology and Psychiatric Sciences appears in each issue of the Journal to stress the role of the epidemiological approach to promote advances in the field of clinical psychopharmacology, with a particular attention to controversial findings. The ultimate aims are to help develop a more critical attitude towards the results of research studies published in the international literature, to promote original research projects with higher methodological standards, and to implement the most relevant results of research in every-day clinical practice. These contributions are written in house by the journal's editorial team or commissioned by the Section Editor (no more than 1000 words, short unstructured abstract, 4 key-words, one Table or Figure and up to ten references).

\title{
Access to mental health services and psychotropic drug use in refugees and asylum seekers hosted in high-income countries
}

\author{
M. Nosè*, G. Turrini and C. Barbui \\ Department of Public Health and Community Medicine, Section of Psychiatry, University of Verona, Verona, Italy
}

In the populations of refugees and asylum seekers hosted in high-income countries, access to mental health care and psychotropic drugs, is a major challenge. A recent Swedish cross-sectional register study has explored this phenomenon in a national cohort of 43403 young refugees and their families from Iraq, Iran, Eritrea, Ethiopia, Somalia and Afghanistan. This register study found lower rates of dispensed psychotropic drugs among recently settled refugees, as compared with Swedish-born residents, with an increase in the use with duration of residence. In this commentary, the results of this survey are discussed in view of their global policy implications for high-income countries hosting populations of refugees and asylum seekers.

Received 11 May 2015; Accepted 15 June 2015; First published online 6 July 2015

Key words: Access, asylum seekers, psychotropic drugs, refugees.

Worldwide, about 44 million people are forcibly displaced because of conflict and persecution, including 15.4 million refugees, 27.5 million internally displaced individuals and over 800000 awaiting resolution of their asylum application. Additionally, the number of people seeking refugee status has progressively increased in the last few years (Tol et al. 2013).

It has been documented that asylum seekers and refugees are a particularly vulnerable group in relation to the development of mental illness, including posttraumatic stress disorder (PTSD) and major depression (Fazel et al. 2005; Steel et al. 2009). Torture experiences

* Address for correspondence: Dr M. Nosè, Department of Public Health and Community Medicine, Section of Psychiatry, University of Verona, Piazzale L.A. Scuro, 10 - 37134 Verona, Italy.

(Email: michela.nose@univr.it) and cumulative exposure to trauma are the strongest factors associated with PTSD and depression, respectively. In addition, numerous resettlement stressors may worsen trauma-related mental health symptoms, including unemployment, unsafe housing, social isolation, discrimination, language and cultural barriers (Silove, 2012). Longitudinal studies of refugees in resettlement confirm that some populations may continue to struggle with high rates of psychiatric illness decades later (Carlsson et al. 2006).

Although healthcare programmes for refugees and asylum seekers consider incorporating a mental health component, there are questions concerning the extent to which refugees are able to properly access mental health services (Crosby et al. 2013). Cultural, structural and psychological barriers to care have been identified. Cultural barriers include lack of understanding of mental health conditions related to trauma, a 
reluctance to initiate conversations about mental health symptoms, and mental health stigma. Structural barriers include language and interpreter difficulties, lack of insurance and cost-related issues. Furthermore, the degree and quality of collaboration between agencies such as refugee reception offices, social and mental health services may influence refugees' access to appropriate care. Psychological barriers include trauma-related symptoms, such as avoidance, that may inhibit discussions of mental health issue (Thomson et al. 2015). Culture also shapes experience and expression of symptoms, signs of mental disorders and evaluation of behaviour. In cross-cultural encounters, signs may thus be misinterpreted. A compelling issue is therefore how the mental health system responds to structural barriers, cultural norms and trauma-related symptoms of refugees and asylum seekers in need of mental health services in high-income countries.

Psychotropic drug use is often used as an indicator of mental health problems in large populations (Barbui, 2015), but its use in refugees and immigrants is very rare in the international literature. Key issues related to psychotropic drug use among refugees have recently been analysed by Brendler-Lindqvist et al., who conducted a cross-sectional register study of a national cohort of 43403 young refugees and their families from Iraq, Iran, Eritrea, Ethiopia, Somalia and Afghanistan and a comparison population of 1.1 million Swedishborn residents (Brendler-Lindqvist et al. 2014). Lower rates of dispensed psychotropic drugs among recently settled refugees, as compared with Swedish-born individuals, were found with an increase in the use with duration of residence. It is reasonable to assume that levels of drug consumption may reflect both mental health status as well as access to care, and Brendler-Lindqvist et al. noted that this pattern of drug use might suggest barriers to access mental health care: high rates of mental disorders in refugees, consistently reported in the literature, do not match with the recorded low rates of dispensed drugs (Brendler-Lindqvist et al. 2014). Thus, it seems reasonable to interpret the low rates of psychotropic drug use as related to on a lower access to care than to a better health status.

Brendler-Lindqvist et al. reported that the use of antipsychotic drugs was similar between different refugee groups and the comparison group of Swedish-born residents. One explanation for this may be that antipsychotic drugs are prescribed for more severe conditions, often involving psychotic symptoms, where the need for psychiatric treatment is more obvious, and often triggered by other people rather than the patient him/herself.

Poor access to psychotropic drugs might be explained by a lack of recognition of mental disorders in the refugee population. As on-going and accumulated distress is very common and expected in this population, it may not receive specific clinical attention unless it evolves into severe psychological and psychiatric problems. Furthermore, the applicability and appropriateness of western concepts of mental illnesses for use in non-western populations is not straightforward, and this may also be related to the difficulties of making a diagnosis. Finally, which measures should be employed to properly assess culturally and linguistically diverse populations is still under debate (Al-Obaidi et al. 2015).

This study further expands the current knowledge on immigrants and mental health services use, and suggests that social, cultural, religious, linguistic, geographic, economic variables, as well as discrimination and stigma, contributes to lower access and use. Access to services has been defined as not only getting to service, but also getting to the right service at right time to promote improved health outcomes' (Peters et al. 2008). Barriers to services are both real and perceived obstacles that prevent or interfere with access to services.

Take-home messages from this survey include the following. First, strategies aimed at improving access and rationale use of psychotropic drugs should receive specific attention, considering that one of the main challenges for mental health services is the need to flexibly adapt to constantly changing population health care needs; this flexibility should be adapted to the local organisation of health care systems, so that different countries may develop different pathways of care to meet similar needs.

Second, studies are needed to further elucidate and identify current barriers to mental health care for refugees and asylum seekers hosted in high-income countries, so that effective measures can be implemented. Interventions that can lower these barriers are needed to enable newly settled refugees to access mental health care and receive correct treatment on equal terms with the native population. In particular, successful referrals to mental health professionals should be improved by better care coordination, establishing trust, resolving access barriers and providing culturally competent care.

Third, epidemiological research focused on understanding current patterns and trends in health utilisation is recommended (Thomson et al. 2015), so that innovative pathways of care, integrated into the existing systems of care, may be planned and developed. It should be noted, in this regard, that several studies have already collected epidemiological and clinical data on mental disorders in refugees and asylum seekers, but most of these studies have been conducted in specific ad hoc services rather than within available systems of public health and mental health care. 
Lastly, inclusion of a mental health component during initial health care assessment might be seen as an optimal strategy to utilise available resources to reduce the burden of psychological distress and mental illness, as we already know that such an approach would represent a powerful aid to diagnosis (AlObaidi et al. 2015), and would also help reduce stigma and increase access to mental health care, including better and more rational use of psychotropic drugs.

\section{Acknowledgements}

None.

\section{Financial support}

No financial support was received for this paper from any funding agency, commercial or not-for-profit sectors.

\section{Conflict of Interest}

None.

\section{References}

Al-Obaidi A, West B, Fox A, Savin D (2015). Incorporating preliminary mental health assessment in the initial healthcare for refugees in New Jersey. Community Mental Health Journal 51, 567-574.

Barbui C (2015). Access and use of psychotropic medicines in low-resource settings. Epidemiology for Clinical

Psychopharmacology 24, 206-209.
Brendler-Lindqvist M, Norredam M, Hjern A (2014). Duration of residence and psychotropic drug use in recently settled refugees in Sweden - a register-based study. International Journal of Equity in Health 13, 122.

Carlsson JM, Mortensen EL, Kastrup M (2006). Predictors of mental health and quality of life in male tortured refugees. Nordic Journal of Psychiatry 60, 51-57.

Crosby SS (2013). Primary care management of non-English speaking refugees who have experienced trauma. Journal of the American Medical Association 310, 519-528.

Fazel M, Wheeler J, Danesh J (2005). Prevalence of serious mental disorder in 7000 refugees resettled in western countries: a systematic review. Lancet 365, 1309-1314.

Peters DH, Garg A, Bloom G, Walker DG, Brieger WR, Rahman MH (2008). Poverty and access to health care in developing countries. Annals of the New York Academy of Sciences 1136, 161-171.

Silove D (2012). Treatment of refugees at the crossroads: the need for an evidence base. Australian and New Zealand Journal of Psychiatry 46, 921-923.

Steel Z, Chey T, Silove D, Marnane C, Bryant R, van Ommeren M (2009). Association of torture and other potentially traumatic events with mental health outcomes among populations exposed to mass conflict and displacement. Journal of the American Medical Association 5, 537-549.

Thomson M, Chaze F, George U, Guruge S (2015). Improving immigrant populations' access to mental health services in Canada: a review of barriers and recommendations. Journal of Immigrant Minority Health March 6 [Epub ahead of print].

Tol WA, Rees SJ, Silove DM (2013). Broadening the scope of epidemiology in conflict-affected settings: opportunities for mental health prevention and promotion. Epidemiology and Psychiatric Sciences 22, 197-203. 